EPJ Web of Conferences 16, 02005 (2011)

DOI: $10.1051 /$ epjconf/20111602005

(C) Owned by the authors, published by EDP Sciences, 2011

\title{
A search for planets around intermediate Mass Stars with the Hobby-Eberly Telescope
}

\author{
M. Adamów ${ }^{1, a}$, S. Gettel ${ }^{2,3}$, G. Nowak ${ }^{1}$, P. Zieliński $^{1}$, A. Niedzielski ${ }^{1}$ \\ and A. Wolszczan ${ }^{2,3}$ \\ ${ }^{1}$ Toruń Centre for Astronomy, Nicolaus Copernicus University, Toruń, Poland \\ ${ }^{2}$ Department for Astronomy and Astrophysics, Pennsylvania State University \\ ${ }^{3}$ Center for Exoplanets and Habitable Worlds
}

\begin{abstract}
We present the discovery of sub-stellar mass companions to three stars by the ongoing Penn State - Torun Planet Search (PTPS) conducted with the $9.2 \mathrm{~m}$ Hobby-Eberly Telescope.
\end{abstract}

\section{INTRODUCTION}

Searches for planets around giant stars extend studies of planetary system formation and evolution to stars substantially more massive than $1 \mathrm{M}_{\odot}$ (Hatzes et al. 2006; Sato et al. 2008; Niedzielski et al. 2009). Although searches for massive sub-stellar companions to early-type stars are possible (Galland 2005), it is much more efficient to utilize the power of the radial velocity (RV) method by exploiting the many narrow spectral lines of GK-giants, the descendants of the main sequence A-F type stars, sufficient to achieve $\mathrm{a}<10 \mathrm{~ms}^{-1} \mathrm{RV}$ measurement precision.

The GK-giant surveys provide constraints on the efficiency of planet formation as a function of stellar mass and chemical composition. In fact, analyses by Johnson et al. (2007) and Lovis \& Mayor (2007) extend to giants the correlation between planetary masses and primary mass that is observed for the lower-mass stars. This is most likely because massive stars tend to have more massive disks. These results are in accord with the core accretion scenario of planet formation (Kennedy \& Kenyon 2008). The steadily extending baselines of the ongoing surveys of post-MS giants should begin furnishing multiplanet system detections that are needed to study the dynamical evolution of planets around the main-sequence stars. The multiplicity of planetary systems around MS stars has been firmly established (Wright et al. 2009) and this tendency is expected to be present among giants as well. In fact, the ongoing search for planets around GK-giants with the Hobby-Eberly Telescope (HET) by our group (Penn StateTorun Planet Search, hereafter PTPS) has already tentatively identified a two-planet system around the K0-giant HD 102272 (Niedzielski et al. 2009), and long-term trends in the RV data have been seen in other surveys (e.g. Sato et al. 2008).

We present the detection of a planet around the K3-giant HD 240210, and show evidence that the star has more low-mass companions, and the intriguing discovery of two brown dwarf-mass bodies orbiting the K2-giant BD +20 2457. We also describe the discovery of a Jupiter-mass planet and a non-linear $\mathrm{RV}$ trend in the K2-dwarf, BD +14 4559.

ae-mail: adamow@astri.umk.pl

This is an Open Access article distributed under the terms of the Creative Commons Attribution-Noncommercial License 3.0, which permits unrestricted use, distribution, and reproduction in any noncommercial medium, provided the original work is properly cited. 


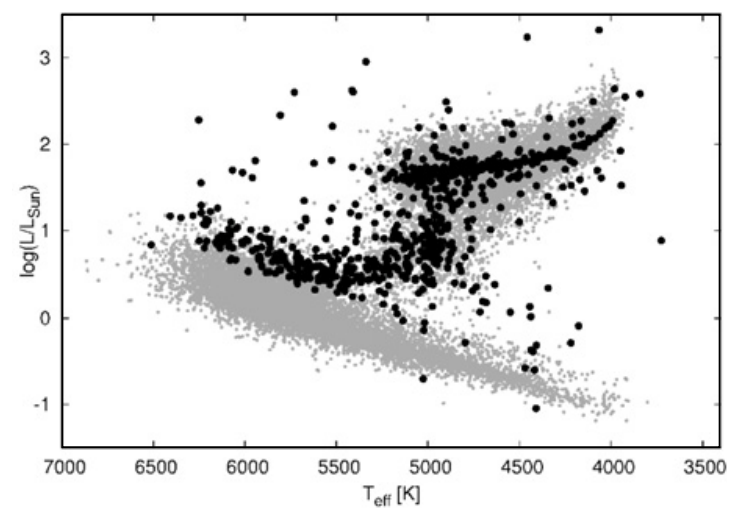

Figure 1. Observed stars: black - stars from red giant clump region; this group contains stars of various masses over a range of evolutionary tracks; intermediate-mass dwarfs, which are about to leave main sequence; giants; grey - stars from Tycho 2 Catalouge not included in the PTPS. Effective temperatures and $\log L / L_{\odot}$ for all groups of stars presented here were obtaind from our compilation of literature photometric indicies and the calibration of parallaxes and proper motions of stars (Adamów 2008).

\section{OBSERVATIONS AND STELLAR PROPERTIES}

Stars observed within PTPS are presented in Fig. 1 (red, green and blue points). Observations were made with the High Resolution Spectrograph of HET with $\mathrm{R}=60$, 000. Radial velocities were measured using the standard $I_{2}$ cell calibration technique (Butler et al. 1996). The radial velocity for each epoch is the mean value of measurements from 17 echelle orders with an intrinsic uncertainty of $6-8 \mathrm{~ms}^{-1}$ at $1 \sigma$ level. The stellar atmospheric parameters were estimated in Zieliński et al. (2009), following Takeda et al. (2005a,b). Stellar masses were derived by fitting $\log \left(L / L_{\odot}\right), \log \left(\mathrm{T}_{e f f}\right), \log (g)$, and metallicity to the evolutionary track of Girardi et al. (2000).

\section{DATA ANALYSIS}

For all three stars, line bisectors and curvature of $\sim 1000$ spectral line profiles show no correlation with radial velocity variations. $H_{\alpha}$ equivalent widths are used as an indicator of chromospheric activity. No significant periodicities are found. Photometric time series from Hipparcos are available for each star, with some additional data from Tycho, NSVS and ASAS, but no significant correlation with radial velocity measurements are found. The absence of such correlations indicates that the observed RV variations are due to orbital motion. Single Keplerian orbit fit by least-squares minimization attempted for each star. Stellar 'jitter' accounted for in K-giants by adding $\sim 20 \mathrm{~ms}^{-1}$ additional error in quadrature. For each star, the single orbit best-fit model leaves non-random trends. The statistical significance of these trends assessed by calculating the false alarm probabilities (FAP) by the radial velocity scrambling method (Wright et al. 2007), for a null hypothesis that the trends can be accounted for by a single Keplerian orbit and noise. FAP $<0.1 \%$ for each, indicating that the additional, systematic RV variations seen in the data are statistically significant. For more detailed data analysis, please see Niedzielski et al. (2009).

\section{SUMMARY}

We report the detections of a new Jupiter-mass planet around a MS star and at least three new substellar companions to GK-giants. K2-dwarf BD +14 4559 has a planet with a minimum mass of $1.5 \mathrm{M}_{\mathrm{J}}$ in a 269-day, 0.79 AU, e $=0.22$ orbit, with a second, long-term trend which is likely to be another planet. This detection is the first result of the extension of the PTPS to evolved dwarfs in the upper envelope 
Research, Science and Technology of Brown Dwarfs and Exoplanets

of the MS. K3-giant HD 240210 is very likely a multiplanet system, though more data will be required to obtain a clear orbital solution. The provisional parameters for one planet that can be fitted for give a $6.9 \mathrm{M}_{\mathrm{J}}$ body in a 501-day, $1.33 \mathrm{AU}, \mathrm{e}=0.14$ orbit that will have to be revised, when another planet (or planets) are added to the current model. K2-giant BD +20 2457 is well modeled by two Keplerian orbits with the periods of 379 and 621 days, 1.4 and 1.9 AU semi-major axes, and the respective eccentricities of 0.14 and 0.18 . For the estimated stellar mass of $2.8 \mathrm{M}_{\odot}$, the minimum masses of the orbiting bodies are 11.6 $\mathrm{M}_{\mathrm{J}}$ and $19.8 \mathrm{M}_{\mathrm{J}}$. The $\sim 1.6$ ratio of the orbital periods in the BD +202457 system places the orbits close to the 3:2 mean motion resonance (MMR), indicating that the two bodies share a common origin from a disk that once surrounded the primary star. The mass of BD +20 2457 is poorly known and spans a range that is at least as wide as $1.3-4.3 \mathrm{M}_{\odot}$. The mass constraints on BD $+202457 \mathrm{~b} \& \mathrm{c}-$ There is a $95 \%$ probability that the inclination of the system is between $18^{\circ}$ and $90^{\circ}$, making masses near the hydrogen burning limit very unlikely. Similarly, it is unlikely that both masses stay below the deuterium burning limit, unless one accepts an unrealistically low primary mass, approaching $1 \mathrm{M}_{\odot}$ or less. The orbital separation and mass range of these substellar companions make it reasonable to assume that they have originated from a massive disk and acquired enough mass to place them above the formal $13 \mathrm{M}_{\mathrm{J}}$ brown dwarf limit. This raises an intriguing possibility that, in the case of substellar-mass companions to giants, both the concept of the brown dwarf desert (Marcy and Butler 2000) and the meaning of the deuterium burning limit will have to be revisited.

We acknowledge the financial support from the Polish Ministry of Science and Higher Education through grant 1P03D-007-30. MA, PZ and GN are recipients of the UE Ph.D. Scholarship Programme ZPORR 2008/2009. GN is a recipient of a graduate stipend of the Chairman of the Polish Academy of Sciences. The Hobby-Eberly Telescope (HET) is a joint project of the University of Texas at Austin, the Pennsylvania State University, Stanford University, Ludwig-Maximilians-Universität München, and Georg-August-Universität Göttingen. The HET is named in honor of its principal benefactors, William P. Hobby and Robert E. Eberly.

\section{References}

[1] Adamów, M. 2008, Determinig Effective Temperature of Evolved Stars - Master's thesis (in Polish);

[2] Butler, R. P.; Marcy, G. W.; Williams, E.; McCarthy, Ch., and Dosanjh, P., PASP 108, (1996) 500-509;

[3] Galland, F.; Lagrange, A.-M.; Udry, S.; Chelli, A.; Pepe, F.; Beuzit, J-L., and Mayor, M., A\&A 444 (2005), L21-L24;

[4] Girardi, L.; Bressan, B.; Bertelli, G., and Chiosi, C., A\&AS 141 (2000), 371;

[5] Hatzes, A. P., et al., A\&A 457 (2006) 335-341;

[6] Johnson, J. A., et al., ApJ 665 (2007), 785;

[7] Johnson, J. A., et al., ApJ, 670 (2007), 833;

[8] Kennedy, G. M., and Kenyon, S. J., ApJ 673 (2008), 502;

[9] Lovis, C., and Mayor, M., A\&A 472 (2007), 657;

[10] Marcy, G. W., and Butler, R. P., PASP 112 (2000), 137;

[11] Niedzielski, A., et al., ApJ 669 (2007), 1354;

[12] Niedzielski, A.; Nowak, G., and Zieliński, P., IAU Symposium 249 (2008), 49;

[13] Niedzielski, A., and Wolszczan, A., 2008, IAU Symposium 249 (2008), 43;

[14] Niedzielski, A., et al., ApJ 707 (2009), 768-777;

[15] Sato, B., et al., PASJ 60 (2008), 539-550;

[16] Takeda, Y., et al., PASJ 57 (2005), 27;

[17] Takeda, Y., et al., PASJ 57 (2005), 109;

[18] Wright, J. T., et al., ApJ 657 (2007), 533;

[19] Wright, J., et al., ApJ 693, 1084;

[20] Zieliński, P., et al., arXiv-0904.0374 (2009). 